from his earliest studies he has developed a faculty for rigorous analysis and for combining different effects. He knows from experience that a problem can only be solved after it has been properly analyzed on the basis of its boundary conditions. From his early training he will also have acquired a broad overall perspective which enables him to spot a large variety of areas of application or to employ a fundamental approach in tackling problems. And more than enough problems are certainly bound to confront us in the coming years.

In this connection I need only draw attention to the efforts that will be needed to solve the energy problem. It is not only necessary to develop new methods of generating and storing energy, ranging from nuclear to solar energy, but to develop new methods and materials which will allow the more efficient utilization of energy at both low and superhigh temperatures, and all must be integrated into an overall system of power economy that is both technically and economically compatible with the existing economic system. At the same time, the current problems of environmental protection and safety demand an overall perspective based on technological and scientific knowledge which reaches far beyond the specialized knowledge of individual experts.

I have here of course concentrated on the problems of the physicists, but these can be solved only in part by physicists, their solution requiring additionally the cooperation of experts with other types of training and of scientists who work not only in the laboratory but also in production, distribution, etc. It is of some interest to note, as shown in the Table, the large percentage of physicists that ultimately find their fulfilment in branches of activity outside R\&D.

Due to the current interaction of different branches of engineering, a knowledge of physics is important not only in research \& development but is equally important in solving problems in production, distribution and patent law.

It goes without saying that the broad spectrum of problems encountered in a steadily advancing technology can only be solved if physicists are prepared to acquire capabilities and knowledge beyond what they have learned at college. Siemens accordingly offers a large variety of refresher courses for the benefit of physicists with their already extensive basic training. Thus it is not surprising that physicists are to be found in responsible positions where new

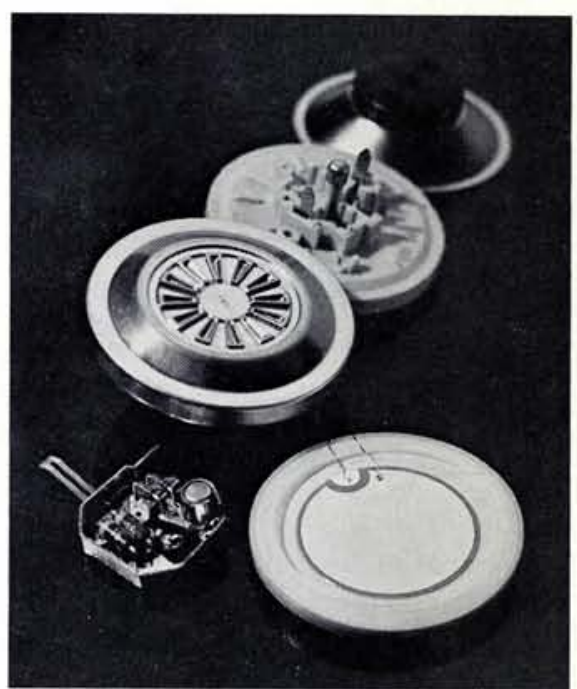

Fig. 2 - Siemens piezo microphone.

types of project need to be managed and a broad knowledge of various different scientific and technological disciplines is essential.

Finally it will be noted from the foregoing that electrical engineering is currently more closely allied with physics than ever before, so that optimal interaction between the two is essential for the whole of the future and consequently also for a vast electrical engineering organization such as the Siemens $A G$ with its worldwide responsibilities.

\title{
The International Laboratory of High Magnetic Fields and Low Temperatures in Wroclaw

The proposal to create the International Laboratory of High Magnetic Fields and Low Temperatures (I.L.) emerged for the first time at a meeting of COMECON experts on low temperature physics in 1964. Subsequently, Wroclaw in Poland was proposed as the site, as the necessary DC power sources already existed there, in the form of a substation of the LowSilesian Power Plant. This station which was presented to the Polish Academy of Sciences comprises several generators with a total capacity of $5.5 \mathrm{MW}$. On 11 May 1968, the representatives of the Bulgarian Academy of Sciences, the Academy of Sciences in the GDR, the Polish Academy of Sciences and the Academy of Sciences of USSR signed the agreement that set up the Laboratory and approved the Statutes and Regulations.

I.L. is a legally independent organi- zation supported by the four Academies and is supervised by a Council on which each is represented by two places. The Chairman of the Council is elected for a term of two years and, on a proposal submitted by the Polish Academy of Sciences, the Director of the I.L. for a term of three years. The Deputy Director is again elected but for a term of two years. The Director is directly responsible for the activity of the I.L. to Council, which each year establishes the budget and the scientific research programme. It also accepts the annual scientific and financial reports of the directorate, the last being examined by a Control Committee on which each Academy has one representative. The chairman is appointed in rotation.

The Council meets twice a year and its resolutions are passed by simple majority, each Academy casting one vote. Since its foundation, the
Chairman of Council has been Professor N.Eu. Aleksejevskii, and the position of Director held by the author. As Deputy Director, Prof. F. Lange (GDR), Dr. K. Berthel (GDR), Dr. Eu Leyarovskii (BDR) and recently $\mathrm{Dr}$. Cz. Bazan (Poland) have acted in turn.

\section{Major Facilities}

It was decided that the first aim should be to make available a steady magnetic field of $20 \mathrm{~T}$ which was achieved progressively with the construction of three Bitter magnets designed by K. Trojnar. The characteristics of these magnets (I-III) are given in the following table:

Field stability is about $10^{-3}$. Using dysprosium or permendur concentrators, it is possible also to increase the field strength by at least $2 \mathrm{~T}$. In parallel, the cooling system was extended and now includes two circuits. The primary closed system contains deionized water at $17 \mathrm{~atm}$ pressure, 
Bitter Magnets Consîructed

\begin{tabular}{llll}
$\begin{array}{l}\text { Fieid } \\
\text { Strength, }\end{array}$ & $\begin{array}{l}\text { Working Space } \\
\text { Diameter, } \mathrm{mm}\end{array}$ & $\begin{array}{l}\text { Electric } \\
\text { Power, } \\
\mathrm{T}\end{array}$ & $\begin{array}{l}\text { Primary Cooling } \\
\text { Water } \\
\text { Circuit, } \mathrm{m}^{3} / \mathrm{h}\end{array}$ \\
10 & 50 & 2,68 & 90 \\
15 & 40 & 4,4 & 145 \\
20 & & 5,67 & 200 \\
7,5 & 30 & 1,34 & 26 \\
7,0 & 85 & 2,37 & 94 \\
5,5 & 180 & 1,96 & 80 \\
\multicolumn{2}{l}{ ists of three equiaxial coils. } & &
\end{tabular}

cooled in heat exchangers by the secondary water that is spray-cooled and made up from our own wells.

Control of the Bitter magnets is separated for safety reasons from the measuring rooms. These are equipped with such instruments as $X-Y$ recorders, temperature controllers, magnetometers and other instruments of high standard necessary for carrying out physical measurements of superconducting and magnetic properties.

Beside the Bitter magnets, the I.L. possesses a superconducting magnet of 15 T ("Intermagnetics") with a working bore of $20 \mathrm{~mm}$ diameter and several other small superconducting magnets fabricated mostly within the Laboratory. Liquid helium is supplied by the Laboratory of Specialised Scientific Equipment in Wroclaw, which is linked to the Institute for Low Temperature and Structure Research of the Polish Academy of Sciences. The I.L. has a close cooperation with this Institute in various fields, particularly for the production of different materials such as alloys and single crystals, as well as their analytical control by electron microprobe and X-ray diffraction. During 1977, the I.L. consumed about 1 GWh of electrical energy and 14000 I of liquid helium.

The I.L. has at its disposal a well equipped mechanical workshop which produce the Bitter discs, the metallic cryostats and other equipment.

\section{Research Programme}

Scientific research is undertaken, according to the plan approved by Council, by the permanent scientific team working in close cooperation with the guest scientists, delegated by the four participant Academies. Amongst the main subjects of investigation carried out in the I.L. one may distinguish the following areas of activity:

1) Construction of Bitter magnets 2) Properties of hard superconductors 3 ) Magnetic materials 4) Galvanomagnetic properties of metals 5) Production of temperatures below $1 \mathrm{~K}$ and measurement of specific heats in this range.

\section{Magnet Development}

The construction of Bitter magnets presents many scientific and technological problems: the influence of holes in the Bitter disc on the current density distribution; the calculation of mechanical stresses limiting the magnetic fields; heat exchange in cooling channels. A reliable safety system protecting the magnets against serious damage in the case of interdisc short-circuits has been elaborated. It is well known that insulation ageing and interdisc contact deterioration cause local overheating, and the damage propagates along the magnet axis.

\section{Hard Superconductors}

A broad programme of research into the behaviour of hard superconductors in high magnetic fields is carried out to determine which physical mechanism is responsible for high critical parameters. In one series the influence of $3 \mathrm{~d}$ metals like $\mathrm{Fe}, \mathrm{Ni}$ and Co impurities on the critical properties of $\mathrm{Nb}_{3} \mathrm{Sn}, \mathrm{Nb}_{3} \mathrm{Al}, \mathrm{V}_{3} \mathrm{Si}, \mathrm{Nb}_{3} \mathrm{Ga}$ was investigated. In a few cases only, e.g. $\mathrm{Fe}$ in $\mathrm{Nb}_{3} \mathrm{Ga}, \mathrm{V}_{3} \mathrm{Si}$ and $\mathrm{Nb}_{3} \mathrm{Sn}$ was the expected drop of the critical temperature and field observed. In general, these impurities behave as if they were non-magnetic, a fact which could have practical importance. The influence of various impurities in vanaalso studied for determining optimal technological parameters for the production of $\mathrm{V}_{3} \mathrm{Ga}$ cables which have the best characteristics for high fields. Similar investigations were carried out for $\mathrm{NbTi}$ as regards the oxygen and nitrogen content.

Intensive research on the behaviour of ternary molybdenum chalcogenides which were recognized by Swiss workers as having the highest critical fields has been conducted. The influence of different substitutes at various concentrations in ternary, and dium and gallium bronzes has been higher order molybdenum chalcogenides and also the influence of ferromagnetic impurities was determined. Iron and nickel cause a sharp drop of the critical temperature and field, whereas for example, europium and ytterbium impurities lead to an increase of the critical field, probably as a result of compensation of the external by the local field (Jaccarino - Peter effect). Partial substitution of lead by tungsten showed an unexpected improvement of superconducting properties.

Nitrides and carbides of niobium, for example $\mathrm{NbCN}$, exhibit also high critical temperatures. In thin films of these materials implantation by nitrogen ions of sufficiently high energies was shown to lead to an increase of the critical temperature. Niobium and titanium hydrides were further subjects of investigation. It was established that a probable reason for the disappearance of superconductivity of $\beta$ niobium hydride is a substantial decrease of electron state densities, manifesting itself in a drop of the electron specific heat coefficient $\gamma$. For the $\mathrm{TI}_{0.75} \mathrm{Nb}_{0.24} \mathrm{H}_{\%}$ system, a decrease of $T_{c}$ and the disappearance of superconductivity was observed for $x>0.9$ at least above $1.7 \mathrm{~K}$. An effort to connect these data with those related to the electron density of states and the Debye temperature has been undertaken on the basis of specific heat measurements at low temperatures. Many other measurements important for the production of superconductors complete this area.

\section{Magnetic Materials}

The main group of materials investigated in high magnetic fields consists of uranium compounds with nonmetals of the $\mathrm{Vb}$ and VIb group. In particular, the following compounds and systems have been examined: $U_{3} X_{4}$ and $U_{3} Y_{4}$ where $X$ and $Y$ are elements of the Vth and VIth group respectively, e.g. solid solutions $U X_{1-x} Y_{x}$ $\beta$-bichalcogenides UY ${ }_{2}$, sesquichalcogenides of the $U_{2} Y_{3}$ type, bichalcogenides like USSe, USTe, USeTe, compounds of the UXY type, mostly in the form of polycrystalline samples.

Bichalcogenides, pnictides of the $\mathrm{U}_{3} \mathrm{X}_{4}$ type and compounds of the UXY type are ferromagnetic, as was established many years ago in the Institute of Low Temperature and Structure Research. Magnetization as a function of the external field was determined and from that the magnetic moment of the uranium ions deduced. Investigations on monocrystals of these 
compounds enabled us to discover their extremely strong anisotropy fields reaching $10^{6} \mathrm{Oe}$. In the case of solid solutions $U X_{1-x} Y_{x}$ we are dealing with competing antiferromagnetic ordering in monopnictides and ferromagnetic ordering in monochalcogenides. Magnetic phase diagrams have been derived which disclose that the high magnetic fields broaden significantly the ferromagnetic region.

Other groups of magnetic materials investigated include the compounds of rare earth metals with elements of the third group of the $\mathrm{RX}_{3}$ and $\mathrm{AuCu}_{3}$ type of structure. For example a metamagnetic transition in $\mathrm{PrSn}_{3}$ at $8.7 \mathrm{~T}$ has been found and a multithreshold transition in Dyln $n_{3}$. Further magnetic research concernes the boroferrites and the double ferromagnetic chromium chalcogenides.

\section{Galvanomagnetic Properties}

An important field of research comprises the galvanomagnetic investigations of the purest metals for determining their electronic structure. The most interesting results were obtained with monocrystals of transition metals like $\mathrm{Nb}, \mathrm{Mo}, \mathrm{Ru}$. Deviations from the quadratic dependence of magnetoresistance as well as thermopower oscillations in niobium were interpreted as a result of magnetic breakdown between an open Fermi surface at the third Brillouin zone and the enclosed sheet of the second zone. Magnetoresistance measurements performed on iron with $\varrho_{293} / \varrho_{4.2}=1500$ showed a quadratic dependence of the resistity increase and oscillations in some directions, which confirmed the assumption that iron is a compensated metal.

Also in molybdenum of very high purity $\left(\varrho_{293} / \varrho_{4.2} \sim 10^{6}\right)$ a quadratic dependence of magnetoresistance up to the highest fields reached has been found. Specific resistivity with applied magnetic fields was higher at $4.2 \mathrm{~K}$ by two orders of magnitude than at room temperature. For ruthenium monocrystals with $\varrho_{293} / \varrho_{4.2} \sim 10^{3}$, measurements of magnetoresistance and thermopower showed oscillations in definite field directions. These effects were explained as a result of magnetic breakdown between an open Fermi surface and a "lens". Magnetoresistance measurements on polycrystalline aluminium wires of various purity reaching $\varrho_{293} / \varrho_{4.2}=3 \times 10^{4}$ showed that in high fields there exists a linear increase of electrical resistivity in all samples. For samples of highest purity an additional increase, caused probably by magnetic breakdown, was observed in effective fields of the order of $10 \mathrm{~T}$. Such an increase was also observed in thin films of $\mathrm{Al}$ and $\mathrm{Ga}$ with $\varrho_{293} / \varrho_{4.2} \sim 30-50$. It seems therefore to be a universal phenomenon of hitherto unknown origin.

\section{Low Temperatures}

For research at temperatures below $4.2 \mathrm{~K}$, a simple system working with helium- 3 in the field of superconducting magnets of $5 \mathrm{~T}$ and $15 \mathrm{~T}$ has recently been constructed. In this temperature and field region, measurements of specific heat of $\mathrm{Nb}-\mathrm{Zr}$ and $\mathrm{Nb}-\mathrm{Ti}-\mathrm{Fe}$ alloys were carried out for suppressing their superconductivity. Such experiments promise to give valuable data relating to the density of states at the Fermi surface. A model of a state with localized spin fluctuations, the so called Schrieffer instability was experimentally tested on $\mathrm{UAI}_{4}$. To reach lower temperatures the adiabatic demagnetization of nuclear and electron paramagnets is now under way. Preliminary experiments with ruby allowed temperatures below $0.1 \mathrm{~K}$ to be achieved. Nuclear demagnetization experiments have been carried out using praseodymium compounds like Prln proposed by Altschuler with initial temperatures produced by $\mathrm{He}-3$.

\section{Publications and Future Plans}

The results of investigations performed in the I.L. are published in the physics journals and also in preprints produced by the I.L. of which so far 36 have appeared.

Future plans provide for the enlargement of the laboratory space and an increase in the electric power. A decision taken by Council and approved by the Academies is to develop a new site outside Wroclaw on the Odra river which will resolve also the problem of water supply. New DC sources from stabilized rectifiers instead of generators are under design. Together with the Institute for Low Temperature and Structure Research, for which new buildings are already under construction, a centre of research in the field of current physical problems in the solid state and at low temperatures will be established.

The I.L. celebrated its 10th anniversary in May with a two-day Symposium. The proceedings are now in press and will be published by the I.L. under the title: "Physical Properties of Solids in High Magnetic Fields". Copies can be ordered through Ars Polona, Box 1001, P-00-950 Warsaw.

\section{University of Utrecht Nuclear Physics Department}

\author{
The Robert J. Van de Graaff \\ laboratory operates an EN \\ Tandem accelerator; a large \\ variety of light- and heavy-ion \\ beams are available.
}

The laboratory has a vacant staff position and seeks an experienced experimental physicist to take major responsibility in the guidance of Ph.D. students in experimental nuclear physics.

Salary, depending on qualification and experience starts at about HFI 60.000 .

For details or applications (with curriculum vitae, bibliography and names of two referees) write to:

\author{
Dr. C. van der Leun, \\ Fysisch Laboratorium, \\ Princetonplein 5, \\ 3508 TA Utrecht,
} The Netherlands. 